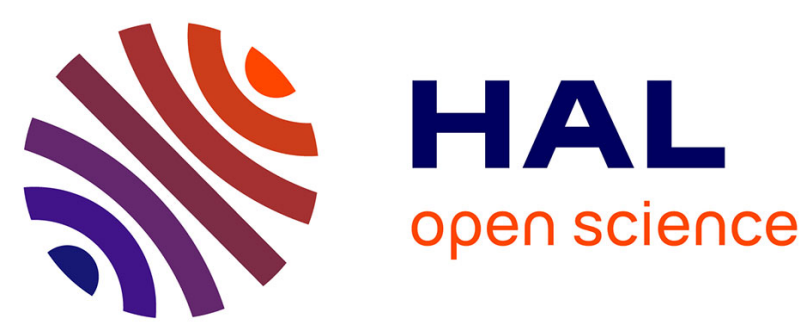

\title{
Ciblage des tissus endométriaux par la $16 \alpha$-[18F]fluoro-17 $\beta$-œstradiol (PET-[18F]FES) : résultats préliminaires dans le diagnostic de l'endométriose
}

\begin{abstract}
A. George, Céline Lefebvre-Lacoeuille, Franck Lacœuille, Pacôme Fosse, Francis Bouchet, Anne Croué, François Hindré, Philippe Descamps, Olivier-François Couturier
\end{abstract}

\section{- To cite this version:}

A. George, Céline Lefebvre-Lacoeuille, Franck Lacœuille, Pacôme Fosse, Francis Bouchet, et al.. Ciblage des tissus endométriaux par la $16 \alpha$-[18F]fluoro-17 $\beta$-œstradiol (PET-[18F]FES) : résultats préliminaires dans le diagnostic de l'endométriose. Médecine Nucléaire - Imagerie Fonctionnelle et Métabolique, 2014, 38 (6), Non spécifié. 10.1016/j.mednuc.2014.04.002 hal-03179476

\section{HAL Id: hal-03179476 \\ https://univ-angers.hal.science/hal-03179476}

Submitted on 24 Mar 2021

HAL is a multi-disciplinary open access archive for the deposit and dissemination of scientific research documents, whether they are published or not. The documents may come from teaching and research institutions in France or abroad, or from public or private research centers.
L'archive ouverte pluridisciplinaire HAL, est destinée au dépôt et à la diffusion de documents scientifiques de niveau recherche, publiés ou non, émanant des établissements d'enseignement et de recherche français ou étrangers, des laboratoires publics ou privés. 


\title{
Ciblage des tissus endométriaux par la $16 \alpha-\left[{ }^{18} \mathrm{~F}\right]$ fluoro- $17 \beta$-œstradiol (PET-[18F]FES) : résultats préliminaires dans le diagnostic de l'endométriose
}

\author{
Effective targeting of endometrial tissue by $16 \alpha-\left[{ }^{18}\right.$ F]fluoro-17ß-œestradiol (PET-[18F]FES): \\ Preliminary results in the diagnosis of endometriosis
}

\author{
A. George ${ }^{\text {a }}$, C. Lefebvre-Lacoeuille ${ }^{\text {b,c }}$, F. Lacoeuille ${ }^{\text {a,c }}$, P. Fosse ${ }^{\text {a }}$, F. Bouchet ${ }^{\text {a }}$, \\ A. Croue ${ }^{\text {d }}$, F. Hindre ${ }^{\text {c }}$, P. Descamps ${ }^{\text {b }}$, O.-F. Couturier ${ }^{a, *, c}$ \\ ${ }^{a}$ Service universitaire de médecine nucléaire, CHU d'Angers, 4, rue Larrey, 49933 Angers cedex 9, France \\ ${ }^{\mathrm{b}}$ Service de gynéco-obstétrique, CHU d'Angers, 4, rue Larrey, 49933 Angers, France \\ ${ }^{\mathrm{C}}$ UMR_S U1066, institut de biologie en santé, IRIS, 4, rue Larrey, 49933 Angers cedex 9, France \\ ${ }^{\mathrm{d}}$ Service d'anatomie pathologique, CHU d'Angers, 4, rue Larrey, 49933 Angers, France \\ Reçu le $1^{\text {er }}$ octobre 2013 ; accepté le 24 avril 2014 \\ Disponible sur Internet le 11 octobre 2014
}

\begin{abstract}
Résumé
Objectifs. - La tomographie par émission de positons (TEP) à la $16 \alpha$-[18F]fluoro- $17 \beta$-œstradiol ([18F]FES) permet d'évaluer in vivo la densité tissulaire en récepteurs aux œstrogènes (RE). Cet article rapporte les premiers cas dans le diagnostic de l'endométriose.

Patientes et méthodes. - Dans le cadre de la mise en place du PHRC ENDOTEP, quatre patientes présentant des signes cliniques et morphologiques (échographie pelvienne \pm IRM) suspects d'endométriose ont bénéficié d'une TEP- $\left[{ }^{18}\right.$ F]FES avant cœlioscopie (analyse macroscopique et analyse histologique et immunohistochimique de l'expression en RE des lésions prélevées).

Résultats. - Chez les quatre patientes, le diagnostic d'endométriose a été retenu macroscopiquement et après analyse histologique d'au moins une lésion. Aucune captation de $\left[{ }^{18} \mathrm{~F}\right] \mathrm{FES}$ par les lésions d'endométriose n'a été observée chez les trois premières patientes sous traitement œstroprogestatif. La quatrième patiente non traitée et en première partie de cycle menstruel lors de la réalisation de la TEP- $\left[{ }^{18} \mathrm{~F}\right] \mathrm{FES}$ présentait en revanche une hyperfixation d'une lésion d'endométriose confirmée en cœlioscopie.

Conclusion. - Nous rapportons le premier cas de fixation de $\left[{ }^{18} \mathrm{~F}\right] \mathrm{FES}$ par une lésion d'endométriose. À travers le projet ENDOTEP, les performances de la TEP- $\left[{ }^{18} \mathrm{~F}\right] \mathrm{FES}$ pour le diagnostic d'endométriose seront évaluées chez des patientes ne recevant pas de traitement hormonal. (C) 2014 Elsevier Masson SAS. Tous droits réservés.
\end{abstract}

Mots clés : Tomographie par émission de positons (TEP) ; 16 $\alpha$-[18F]fluoro-17ß-œstradiol (FES) ; Récepteurs aux œstrogènes ; Endométriose ; Endomètre

\begin{abstract}
Objectives. - Positron emission tomography with $16 \alpha$-[18F]fluoro-17 $\beta$-œstradiol (PET-[18F]FES) is used to evaluate the in vivo tissue density in estrogen receptor (ER). We report here the first cases in the diagnosis of endometriosis.

Patients and methods. - Four patients with clinical and morphological signs (pelvic ultrasound \pm MRI) consistent with endometriosis were enrolled in this National Grant PHRC ENDOTEP. They underwent a PET- $\left[{ }^{18}\right.$ F]FES scan before laparoscopy (macroscopic and histological analysis, immuno-histochemical ER expression).

Results. - In the four patients, the diagnosis of endometriosis was confirmed on the typical macroscopic appearance of laparoscopy plus histological analysis of at least one lesion. No uptake of $\left[{ }^{18} \mathrm{~F}\right] \mathrm{FES}$ by endometriosis lesions was observed in the first three patients with estrogenprogestin therapy. The fourth patient, untreated and in the first part of the menstrual cycle when performing PET- $\left[{ }^{18}\right.$ F]FES, had however an increased $\left[{ }^{18} \mathrm{~F}\right] \mathrm{FES}$ uptake of one lesion of endometriosis confirmed by laparoscopy.
\end{abstract}

\footnotetext{
* Auteur correspondant.

Adresse e-mail : ocouturier70@me.com (O.F. Couturier).
} 
Conclusion. - We report here the first case of $\left[{ }^{18} \mathrm{~F}\right] \mathrm{FES}$ uptake by an endometriosis lesion. With the ENDOTEP trial, the performance of PET-

$\left[{ }^{18} \mathrm{~F}\right] \mathrm{FES}$ for the diagnosis of endometriosis will be evaluated in patients who are not receiving hormone therapy.

(C) 2014 Elsevier Masson SAS. All rights reserved.

Keywords: Positron emission tomography (PET); 16 $\alpha-[18 \mathrm{~F}]$ fluoro-17 $\beta$-œstradiol ([ $\left.\left.{ }^{18} \mathrm{~F}\right] \mathrm{FES}\right)$; Estrogen receptor; Endometriosis; Endometrium

\section{Introduction}

Parmi les modèles d'interaction avec les tissus des médicaments radiopharmaceutiques (MRP), le plus connu est le modèle transporteur GLUT1-[ $\left.{ }^{18} \mathrm{~F}\right] \mathrm{FDG}$ utilisé en tomographie par émission de positons (TEP). D'autres MRP ont été développés ou sont encore en cours de développement pour pallier les insuffisances du $\left[{ }^{18} \mathrm{~F}\right] \mathrm{FDG}$, en particulier son manque de spécificité vis-à-vis du tissu tumoral. Parmi ces autres traceurs, certains reposent sur l'affinité d'un MRP pour un récepteur spécifique (modèle ligand-récepteur) et ouvrent de très nombreuses possibilités, que ce soit dans le cadre de l'immuno-TEP (modèle antigène-anticorps), d'interactions avec des récepteurs couplés à la protéine $\mathrm{G}$ (récepteurs de la somatostatine, bombésine, gastrin-releasing peptide...), du ciblage de l'intégrine alpha-v-béta-3 ou de récepteurs hormonaux.

La TEP à la $16 \alpha-\left[{ }^{18} \mathrm{~F}\right]$ fluoro- $17 \beta$-œstradiol ([ $\left.\left.{ }^{18} \mathrm{~F}\right] \mathrm{FES}\right)$ repose sur le modèle ligand-récepteur, où la $\left[{ }^{18} \mathrm{~F}\right] \mathrm{FES}$ se fixe sur les récepteurs aux estrogènes (RE) intracellulaires. La TEP$\left[{ }^{18} \mathrm{~F}\right] \mathrm{FES}$ est une nouvelle modalité d'imagerie moléculaire permettant d'évaluer in vivo la richesse des tissus en RE actifs [1]. Les pathologies du sein et de l'endomètre sont les principaux modèles explorés en TEP- $\left[{ }^{18} \mathrm{~F}\right] \mathrm{FES}$, en rapport avec leur expression en RE [2-4]. Le principal intérêt de la TEP$\left[{ }^{18} \mathrm{~F}\right] \mathrm{FES}$ semble résider dans sa capacité à caractériser l'expression des RE au sein de l'organisme en un seul examen non invasif, ce qui représenterait une alternative non invasive et plus exhaustive aux biopsies, notamment à distance du bilan initial et lors de maladie multifocale ou métastatique en rechute [5].

Dans le cancer du sein, l'intensité de captation de la $\left[{ }^{18} \mathrm{~F}\right] \mathrm{FES}$ en TEP reflète la densité tissulaire des RE, ce qui permet d'établir le statut RE de la tumeur primitive et de ses éventuelles métastases [6]. L'intensité de captation de la $\left[{ }^{18} \mathrm{~F}\right] \mathrm{FES}$ semble également prédictive de la réponse aux traitements anti-hormonaux [7,8]. En sélectionnant les tumeurs $\mathrm{RE}+$, candidates à la thérapie anti-hormonale, puis en évaluant précocement la réponse thérapeutique, la TEP- $\left[{ }^{18} \mathrm{~F}\right] \mathrm{FES}$ s'inscrit dans une réelle approche «théranostique » $[9,10]$.

Les données de la littérature sur la TEP- $\left[{ }^{18} \mathrm{~F}\right] \mathrm{FES}$ dans l'exploration des pathologies de l'endomètre sont moins nombreuses. Les premières études publiées semblent montrer que la TEP- $\left[{ }^{18} \mathrm{~F}\right] \mathrm{FES}$ pourrait permettre de visualiser les tumeurs bénignes et malignes de l'endomètre et mesurer leur densité en RE comme dans les tumeurs du sein, les pathologies bénignes de l'endomètre exprimant le plus fortement les RE et fixant le plus fortement également la $\left[{ }^{18} \mathrm{~F}\right] \mathrm{FES}$ [11]. Notre équipe s'intéresse à ce nouveau traceur qui n'a pas d'autorisation de mise sur le marché (AMM) en France à travers plusieurs projets de recherche précliniques et cliniques, notamment dans l'endométriose. Cet article rapporte les premiers cas de TEP- $\left[{ }^{18} \mathrm{~F}\right] \mathrm{FES}$ dans l'endométriose.

\section{Patientes et méthodes}

\subsection{Projet ENDOTEP}

Le programme hospitalier de recherche clinique ENDOTEP $^{\circledR}$ (PHRC interrégional 2010) est une étude prospective ouverte multicentrique ayant pour objectif principal d'évaluer les performances de la TEP- $\left[{ }^{18} \mathrm{~F}\right] \mathrm{FES}$ pour établir le diagnostic d'endométriose, en comparaison à l'histologie (examen de référence) des prélèvements de la cœlioscopie. La population étudiée regroupe des femmes prises en charge pour une suspicion d'endométriose et pour lesquelles l'indication d'une première cœlioscopie est déjà posée. Les objectifs secondaires de l'étude sont de comparer les performances de la TEP$\left[{ }^{18} \mathrm{~F}\right] \mathrm{FES}$ à celles des autres techniques d'imagerie (échographie pelvienne et/ou IRM), de comparer la cartographie lésionnelle établie par la TEP- $\left[{ }^{18} \mathrm{~F}\right] \mathrm{FES}$ à celle établie sous cœlioscopie, d'évaluer la corrélation entre l'intensité de fixation de la $\left[{ }^{18} \mathrm{~F}\right] \mathrm{FES}$ et la quantification de l'expression des RE par immunohistochimie, d'évaluer la biodistribution de la $\left[{ }^{18} \mathrm{~F}\right] \mathrm{FES}$ sur l'imagerie TEP et d'évaluer la toxicité éventuelle de la $\left[{ }^{18} \mathrm{~F}\right] \mathrm{FES}$, molécule sans AMM.

Le schéma de l'étude inclut la réalisation de la TEP$\left[{ }^{18} \mathrm{~F}\right] \mathrm{FES}$ lors du même cycle menstruel que la cœlioscopie, en dehors de la période menstruelle et avant le $15^{\mathrm{e}}$ jour du cycle menstruel. La réalisation de la cœlioscopie doit s'effectuer en insu des résultats de la TEP- $\left[{ }^{18} \mathrm{~F}\right] \mathrm{FES}$. Le projet de recherche ne modifie pas les stratégies diagnostiques et thérapeutiques habituelles (en dehors de la réalisation de la TEP- $\left[{ }^{18} \mathrm{~F}\right] \mathrm{FES}$ et de l'examen immunohistochimique fait sur les prélèvements). La réalisation des autres examens d'imagerie (échographie pelvienne, IRM) n'est pas imposée et reste à l'appréciation de chaque praticien.

\subsection{Patientes}

Quatre patientes, âgées de 25, 26, 31 et 35 ans, présentant des signes cliniques (douleurs pelviennes, dysménorrhée, dyspareunie, infertilité) et morphologiques (échographie pelvienne \pm IRM) suspects d'endométriose ont été incluses prospectivement dans le protocole de l'étude ENDOTEP après obtention de leur consentement éclairé (Tableau 1). Trois de ces quatre patientes (trois premières) recevaient un traitement hormonal (traitement médical de l'endométriose) au moment 
Tableau 1

Caractéristiques cliniques des patientes.

Clinical characteristics of patients.

\begin{tabular}{|c|c|c|c|c|}
\hline Patiente no. & $\hat{\text { Age }}$ & Symptômes & Traitement hormonal reçu & Bilan d'imagerie \\
\hline 1 & 35 & $\begin{array}{l}\text { Algies pelviennes chroniques, } \\
\text { douleurs mictionnelles, } \\
\text { dysménorrhées et dyspareunies } \\
\text { résistantes au traitement médical }\end{array}$ & $\begin{array}{l}\text { Estroprogestatif en continu } \\
\text { depuis } 6 \text { mois }\end{array}$ & $\begin{array}{l}\text { IRM et échographie pelviennes : nodule } \\
\text { d'endométriose }(20 \mathrm{~mm}) \text { accolé à la } \\
\text { partie postérieure de la vessie }\end{array}$ \\
\hline 2 & 25 & $\begin{array}{l}\text { Algies pelviennes chroniques, } \\
\text { douleurs à l'exonération, } \\
\text { dysménorrhées et dyspareunies } \\
\text { résistantes au traitement médical }\end{array}$ & Estroprogestatif en continu depuis 1 an & $\begin{array}{l}\text { IRM et échographie pelviennes : nodule } \\
(11 \mathrm{~mm}) \text { d'endométriose au niveau du } \\
\text { ligament utéro-sacré gauche }\end{array}$ \\
\hline 3 & 26 & Infertilité > 1 an, dysménorrhées & Progestatifs en continu depuis 2 mois & $\begin{array}{l}\text { IRM et échographie pelviennes : } \\
\text { endométriome ovarien gauche }(4 \mathrm{~cm}) \text {, } \\
\text { nodules d'endométriose vésicale } \\
(2,5 \mathrm{~cm}) \text {, du cul-de-sac vaginal } \\
\text { postérieur et des ligaments utéro-sacrés }\end{array}$ \\
\hline 4 & 31 & Algies pelviennes, infertilité $>3$ ans & Aucun & $\begin{array}{l}\text { Échographie pelvienne : endométriome } \\
\text { de } 6 \mathrm{~cm} \text { de l'ovaire droit }\end{array}$ \\
\hline
\end{tabular}

de l'inclusion et de la réalisation de la TEP- $\left[{ }^{18} \mathrm{~F}\right] \mathrm{FES}$. Deux étaient traitées par œstroprogestatifs en continu depuis, respectivement, 6 et 12 mois et la troisième était sous progestatifs depuis 2 mois. La dernière et quatrième patiente n'avait pas reçu de traitement hormonal depuis plus d'un an.

\subsection{Imagerie TEP- $\left[{ }^{18} \mathrm{~F}\right] \mathrm{FES}$}

Après un jeûne minimal de $6 \mathrm{~h}$, les patientes ont reçu une injection IV de $4 \mathrm{MBq} / \mathrm{kg}$ de $\left[{ }^{18} \mathrm{~F}\right] \mathrm{FES}$ (laboratoires Cyclopharma Tours, France). L'injection était immédiatement suivie d'une acquisition dynamique de 20 minutes centrée sur le pelvis, puis d'une acquisition précoce débutant en moyenne 25 min (minimum-maximum : 22-34 min) après l'injection et d'une acquisition tardive débutant en moyenne $61 \mathrm{~min}(60$ $63 \mathrm{~min}$ ) après l'injection, toutes les deux centrées sur la région abdomino-pelvienne avec une durée de 2 min $30 \mathrm{sec}$ par pas. Les images étaient réalisées sur un appareil TEP/TDM GE Discovery ST (General Electric GE, Buck, France). L'acquisition TEP était précédée d'une acquisition tomodensitométrique (TDM 8 barrettes, $120 \mathrm{kV}, 64 \mathrm{mAs}, 3,75 \mathrm{~mm}$ d'épaisseur de coupe) pour la correction d'atténuation et le repérage anatomique grâce à la fusion des images TEP et TDM. Les images ont été analysées par des médecins nucléaires expérimentés sur une console d'interprétation Imagys (Keosys, France) avant la transmission des résultats anatomopathologiques et immunohistochimiques. Tout foyer de fixation persistant sur les trois acquisitions et ne correspondant pas à une fixation physiologique de la FES a été considéré comme un foyer d'endométriose. L'intensité de captation de l'utérus et des lésions d'endométriose ont été quantifiées par mesure des SUV max, SUV peak (SUV moyenne la plus intense déterminée dans une sphère de $1 \mathrm{~cm}^{3}$ ) et SUV moyen (défini par un isocontour à $41 \%$ du SUV peak) au sein de régions d'intérêt supérieures à $1 \mathrm{~cm}^{3}$. Les intensités de captation des différents organes (foie, vésicule biliaire, intestin grêle, côlon, vessie) ont été quantifiées par mesure du SUV max et les valeurs moyenne, minimale et maximale de SUV max de chaque organe ont été calculées. L'activité circulante et la captation non spécifique du traceur (bruit de fond) ont été estimées grâce à une région d'intérêt volumique placée sur les tissus musculaires.

\subsection{Chirurgie et analyse anatomo-pathologique}

Les patientes $\mathrm{n}^{\circ} 1$ à 4 ont été explorées en colioscopie au bloc opératoire par un chirurgien gynécologique expérimenté, respectivement, 9, 2, 17 et 7 jours après l'examen TEP$\left[{ }^{18} \mathrm{~F}\right] \mathrm{FES}$. La coelioscopie avait pour objectif de réaliser une cartographie de l'ensemble des lésions d'endométriose et de réaliser au minimum une biopsie lésionnelle pour confirmation histologique. En fonction de chaque situation clinique (patiente prise en charge pour infertilité ou pour symptomatologie douloureuse), une exérèse partielle ou totale des lésions retrouvées a été réalisée.

Les lésions prélevées ont été analysées en immunohistochimie (IHC) à la recherche de récepteurs aux œstrogènes de type $\alpha(\mathrm{RE} \alpha)$. Une lésion était considérée RE+ en IHC si plus de $10 \%$ des noyaux étaient marqués.

\section{Résultats}

\section{1. Étalon de référence : analyse histologique et immunohistochimique}

Chez les quatre patientes, le diagnostic d'endométriose a été confirmé histologiquement sur au moins une biopsie lésionnelle conformément aux recommandations du Collège national des gynécologues obstétriciens français. Conformément à ces mêmes recommandations, l'exérèse chirurgicale n'a pas été réalisée pour certaines des lésions d'endométriose quand cette exérèse ne rentrait pas dans la prise en charge thérapeutique des patientes (patientes infertiles devant être prise en charge par fécondation in vitro). L'analyse histologique n'a donc pas été réalisée sur toutes les lésions visibles en cœlioscopie, mais l'aspect macroscopique de ces lésions étant typique et le diagnostic histologique d'endométriose étant posé chez les quatre patientes, toutes les lésions macroscopiquement typiques ont été considérées de nature endométriosique. 
Tableau 2

Résultats chirurgicaux, histologiques et d'imagerie.

Surgical, histological and imaging findings.

\begin{tabular}{|c|c|c|c|c|c|}
\hline $\begin{array}{l}\text { Patiente } \\
\text { no. }\end{array}$ & Lésions cœlioscopiques & Histo & $\mathrm{IHC}$ & TEP-FES & $\begin{array}{l}\text { Imagerie } \\
\text { morphologique }\end{array}$ \\
\hline \multirow[t]{3}{*}{1} & Lésions d'endométriose superficielle de la fossette ovarienne droite & + & Marquage de $80 \%$ des noyaux & - & - \\
\hline & Lésion d'endométriose superficielle du ligament utéro-sacré gauche & + & & - & - \\
\hline & Nodule d'endométriose de $2 \mathrm{~cm}$ de l'espace vésico-utérin & + & & - & + \\
\hline \multirow[t]{2}{*}{2} & $\begin{array}{l}\text { Lésion d'endométriose profonde de } 11 \mathrm{~mm} \text { du ligament utéro-sacré } \\
\text { gauche }\end{array}$ & + & Marquage de 30 à $50 \%$ des noyaux & - & + \\
\hline & $\begin{array}{l}\text { Lésion d'endométriose péritonéale superficielle de l'espace } \\
\text { vésico-utérin }\end{array}$ & + & & - & - \\
\hline \multirow[t]{4}{*}{3} & Endométriome de $5 \mathrm{~cm}$ de l'ovaire gauche & + & Marquage hétérogène, de 0 à $60 \%$ & - & + \\
\hline & Nodule d'endométriose vésical de $2 \mathrm{~cm}$ & NR & NR & - & + \\
\hline & Lésions d'endométriose du cul-de-sac vaginal postérieur & NR & NR & - & + \\
\hline & Lésions d'endométriose du cul-de-sac vésico-utérin & NR & NR & - & - \\
\hline \multirow[t]{2}{*}{4} & Endométriome de $6 \mathrm{~cm}$ de l'ovaire droit & + & Marquage d'environ $20 \%$ des noyaux & - & + \\
\hline & Lésion profonde rétro-utérine gauche & NR & NR & + & - \\
\hline
\end{tabular}

Histo : histologie ; IHC : immunohistochimie ; NR : non réalisé (lésions non prélevées).

Les foyers d'endométriose, localisés au sein de tissus de soutien de type fibro-musculaire, étaient mesurés au maximum à $1 \mathrm{~mm}$ de grand axe. L'étude immunohistochimique était en faveur d'une expression variable et hétérogène $(0$ à $80 \%)$ des RE $\alpha$ au sein de l'ensemble des lésions d'endométriose (Tableau 2).

\subsection{Biodistribution de la $\left[{ }^{18} F\right] F E S$}

L'intensité de fixation de la $\left[{ }^{18} \mathrm{~F}\right] \mathrm{FES}$ a été gradée en 4 niveaux selon l'échelle visuelle définie de la manière suivante : le niveau 1 correspond à l'intensité de fixation musculaire (bruit de fond), le niveau 2 à une fixation
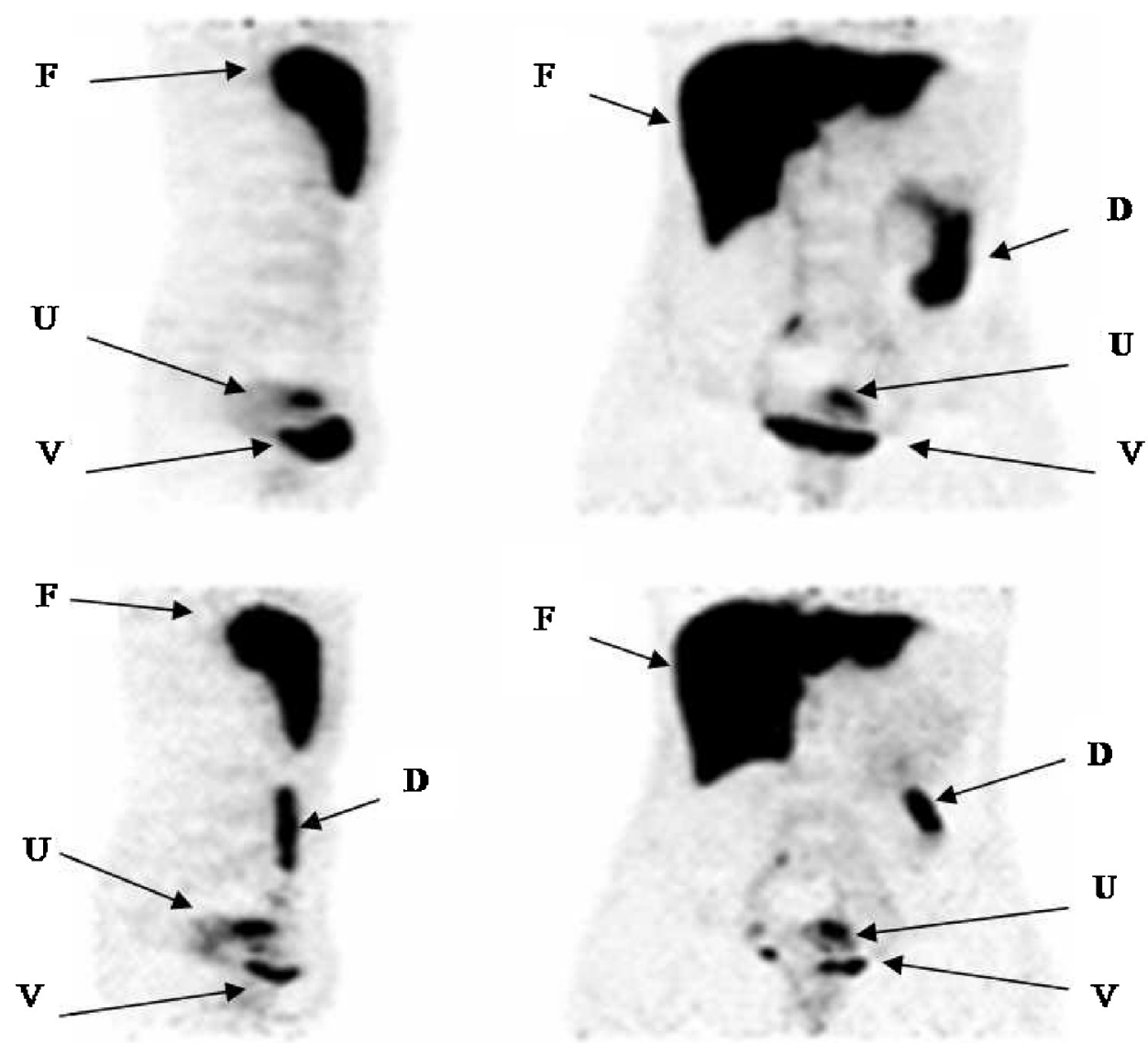

Fig. 1. Biodistribution de la $\left[{ }^{18} \mathrm{~F}\right] \mathrm{FES}$ (patiente $\mathrm{n}^{\mathrm{o}} 4$ ). Coupes sagittales (à gauche) et coronales (à droite) aux temps précoce (en haut) et tardif (en bas). Fixations hépatiques $(\mathrm{H})$ et intestinales $(\mathrm{I})$ intenses, élimination urinaire du traceur au niveau des uretères et de la vessie $(\mathrm{V})$ et captation physiologique marquée du $\left[{ }^{18} \mathrm{~F}\right] \mathrm{FES}$ par l'utérus (U).

Biodistribution of ${ }^{18}$ F]FES (patient 4). Sagittal (left) and coronal (right) slices at early (top) and late (bottom) time. Liver (H) and intestinal (I) intense uptake, urinary excretion of the tracer in the ureters and bladder $(V)$, and marked physiological uterine uptake $(U)$. 
Tableau 3

Intensité de fixation de l'utérus en TEP-FES.

Uterine uptake intensity in FES-PET.

\begin{tabular}{|c|c|c|c|c|c|c|c|c|c|}
\hline \multirow[t]{2}{*}{ Patiente } & \multicolumn{3}{|c|}{ Temps dynamique } & \multicolumn{3}{|c|}{ Temps précoce } & \multicolumn{3}{|l|}{ Temps tardif } \\
\hline & SUV max & SUV peak & SUV moy & SUV max & SUV peak & SUV moy & SUV max & SUV peak & SUV moy \\
\hline 1 & 2,25 & 2,10 & 1,54 & 3,84 & 3,21 & 2,25 & 6,35 & 3,89 & 2,79 \\
\hline 2 & 2,98 & 2,78 & 1,83 & 3,42 & 2,56 & 2,05 & 3,33 & 2,46 & 1,87 \\
\hline 3 & 2,98 & 2,78 & 1,95 & 5,15 & 3,92 & 2,88 & 4,74 & 3,51 & 2,65 \\
\hline 4 & 3,69 & 3,31 & 2,48 & 6,75 & 5,77 & 4,13 & 7,36 & 6,25 & 4,45 \\
\hline Moyenne \pm DS & $2,98 \pm 0,59$ & $2,74 \pm 0,50$ & $1,95 \pm 0,39$ & $4,79 \pm 1,50$ & $3,87 \pm 1,39$ & $2,83 \pm 0,94$ & $5,45 \pm 1,78$ & $4,03 \pm 1,60$ & $2,94 \pm 1,08$ \\
\hline
\end{tabular}

intermédiaire entre les niveaux 1 et 3, le niveau 3 à l'intensité de fixation hépato-intestinale et le niveau 4 à l'intensité de fixation biliaire. Sur les 4 examens TEP- $\left[{ }^{18} \mathrm{~F}\right] \mathrm{FES}$, une captation utérine de la $\left[{ }^{18}\right.$ F]FES est visualisée (Fig. 1). L'intensité de fixation utérine de chaque patiente, de niveau 2 selon l'échelle visuelle, est donnée dans le Tableau 3. Cette fixation s'intensifie globalement avec le temps de captation, avec un SUV max moyen égal à 2,98 $\pm 0,59$ au temps dynamique, 4,79 $\pm 1,50$ au temps précoce et $5,45 \pm 1,78$ au temps tardif. Les fixations physiologiques hépatiques, biliaires et intestinales sont visualisées (SUV max moyens respectifs au temps tardif : $18,24 \pm 2,15,216,20 \pm 62,67$ et $27,56 \pm 15,29$ ), ainsi que l'élimination urinaire du radiotraceur au niveau des reins, des uretères et de la vessie. Une faible fixation de la $\left[{ }^{18} \mathrm{~F}\right] \mathrm{FES}$, de niveau 1 selon l'échelle visuelle, est visualisée au niveau du côlon, des muscles, du myocarde (partiellement visualisée sur les coupes abdominales supérieures), de la rate, de l'estomac et du squelette. Les valeurs moyennes, minimales et maximales de SUV max par organe sont données dans le Tableau 4.

\subsection{Lésions d'endométriose en TEP- $\left[{ }^{18}\right.$ F]FES versus imagerie conventionnelle}

Les différentes lésions d'endométriose diagnostiquées en cœlioscopie sont décrites dans le Tableau 2. Il n'est pas visualisé de foyer d'endométriose en TEP- $\left[{ }^{18} \mathrm{~F}\right] \mathrm{FES}$ chez les patientes $n^{\circ} 1$ à 3 , toutes sous traitement hormonal. Sur les neuf lésions d'endométriose comptées en cœlioscopie chez ces trois patientes, quatre n'avaient pas été visualisées sur le bilan d'imagerie morphologique pré-opératoire (échographie et IRM). Deux lésions d'endométriose utéro-sacrées décrites en

Tableau 4

Intensité de fixation tissulaire en TEP-FES.

FES-PET tissue uptake intensity.

\begin{tabular}{lcccccrrr}
\hline Tissu/organe & \multicolumn{2}{c}{ SUV max (temps précoce) } & & \multicolumn{3}{c}{ SUV max (temps tardif) } \\
\cline { 2 - 3 } & Moyenne & Min & Max & & Moyenne & Min & Max \\
\hline Foie & 20,98 & 18,8 & 25,38 & & 18,24 & 16,26 & 20,31 \\
Vésicule & 151,22 & 17,56 & 365,62 & & 216,20 & 161,73 & 305,64 \\
Grêle & 13,77 & 12,6 & 14,56 & & 27,56 & & 17,53 & 50,34 \\
Colon & 1,09 & 0,86 & 1,48 & & 1,10 & & 0,84 & 1,31 \\
Reins & 2,8 & 2,3 & 3,11 & & 3,17 & & 2,78 & 3,65 \\
Vessie & 39,2 & 9,35 & 74,17 & & 46,33 & & 23,45 & 97,47 \\
Muscle & 1,02 & 0,83 & 1,24 & & 0,84 & & 0,65 & 1,12 \\
\hline
\end{tabular}

Min : valeur minimale ; Max : valeur maximale.
IRM n'ont pas été confirmées en cœlioscopie chez la patiente $\mathrm{n}^{\mathrm{o}} 3$.

Chez la patiente $\mathrm{n}^{\circ} 4$, qui n'était pas sous traitement hormonal, un foyer d'endométriose est visualisé en TEP$\left[{ }^{18} \mathrm{~F}\right] \mathrm{FES}$ sous forme d'un foyer hyperfixant de niveau 2 selon l'échelle visuelle en région pelvienne postérieure gauche sur les acquisitions dynamique, précoce et tardive (SUV max respectifs : 2,2, 3,1 et 3,9) (Fig. 2). Ce foyer correspondait en coelioscopie à une lésion d'endométriose rétro-utérine gauche de $2 \mathrm{~cm}$ (Fig. 3), non visualisée sur l'échographie préopératoire : cette lésion est considérée « vrai-positif » en TEP$\left[{ }^{18} \mathrm{~F}\right] \mathrm{FES}$ et « faux négatif » en échographie pelvienne endovaginale.

Une lésion d'allure kystique hypofixante avec un liseré normofixant en périphérie est visualisée, respectivement, en région ovarienne gauche et droite sur les TEP- $\left[{ }^{18} \mathrm{~F}\right] \mathrm{FES}$ des patientes $n^{\circ} 3$ et 4 . Ces lésions correspondant en cœlioscopie et sur le bilan d'imagerie morphologique à des endométriomes (lésions d'endométriose ovarienne) ont été considérées « faux négatifs » en TEP- $\left[{ }^{18}\right.$ F]FES (Fig. 4).

\section{Discussion}

Jusqu'à présent, l'imagerie TEP-[ $\left.{ }^{18} \mathrm{~F}\right] \mathrm{FES}$ a essentiellement été étudiée dans le cancer du sein et, secondairement, dans les pathologies utérines, le cancer ovarien et les méningiomes $[4,12,13]$. Plusieurs études ont montré une excellente corrélation entre la fixation de la $\left[{ }^{18} \mathrm{~F}\right] \mathrm{FES}$ en TEP et la densité en RE in vitro au sein des lésions primitives ou métastatiques de néoplasie mammaire $[2,3,9]$. La TEP- $\left[{ }^{18} \mathrm{~F}\right] \mathrm{FES}$ pourrait être un outil intéressant pour évaluer le statut RE des patientes, notamment lorsque l'expression tissulaire en RE est hétérogène, lors de tumeurs multiples ou difficilement accessibles, dont les biopsies entraînent une morbidité non négligeable, ou au cours de l'évolution métastatique des néoplasies, où de nouveaux clones tumoraux présentant un statut RE différent de la tumeur initiale peuvent apparaître $[5,14,15]$. Ces changements phénotypiques ont été observés chez 20 à $35 \%$ des patientes avec cancer du sein métastatique [4]. Plusieurs auteurs ont également étudié la place de la TEP- $\left[{ }^{18} \mathrm{~F}\right] \mathrm{FES}$ dans l'évaluation thérapeutique après traitement hormonal $[5,7,10,16,17]$. Les patientes répondeuses au tamoxifène (anti-œstrogène) présentaient un rehaussement métabolique en TEP- $\left[{ }^{18} \mathrm{~F}\right] \mathrm{FDG}$, ou metabolic flare, s'accompagnant d'une diminution de fixation des lésions en 

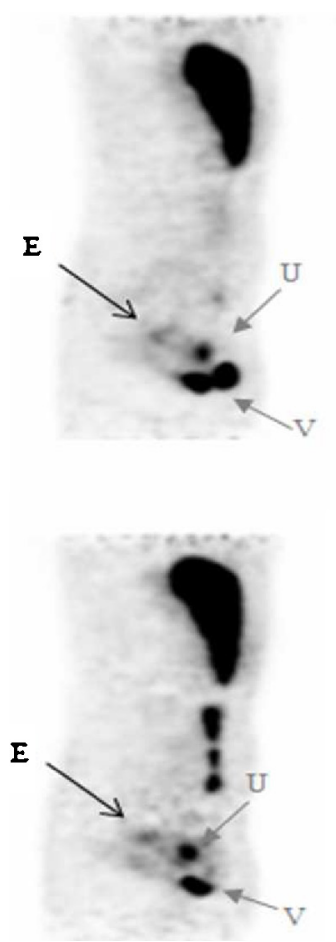
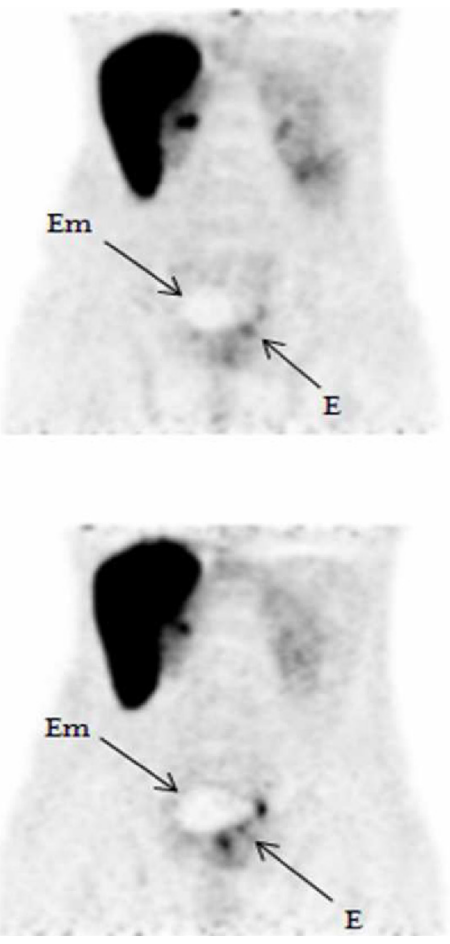
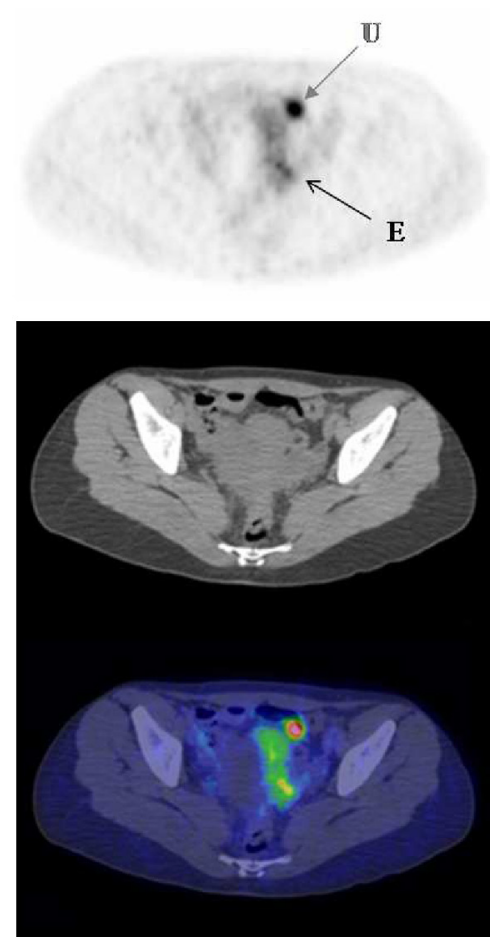

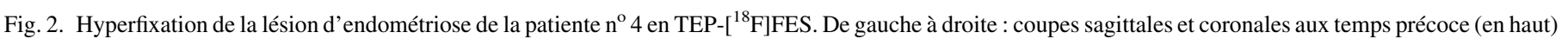

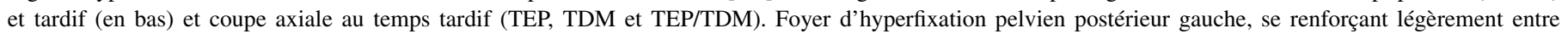

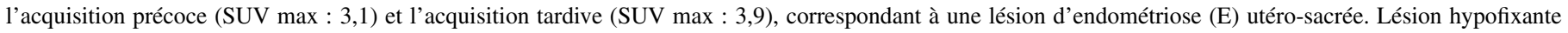

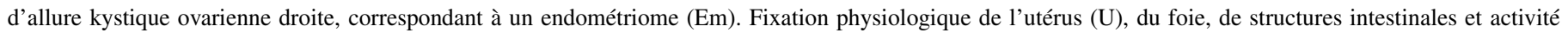
urinaire au sein de la vessie $(\mathrm{V})$.

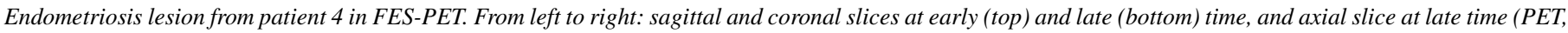

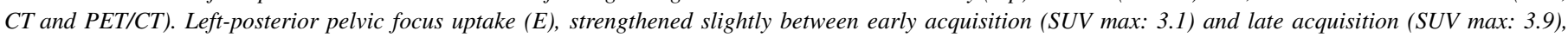

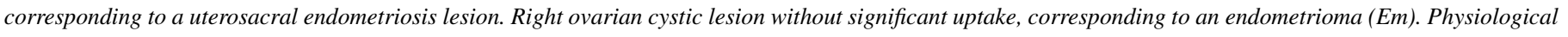
uterine $(U)$, liver and intestinal structures uptake and urinary activity in the bladder (V).

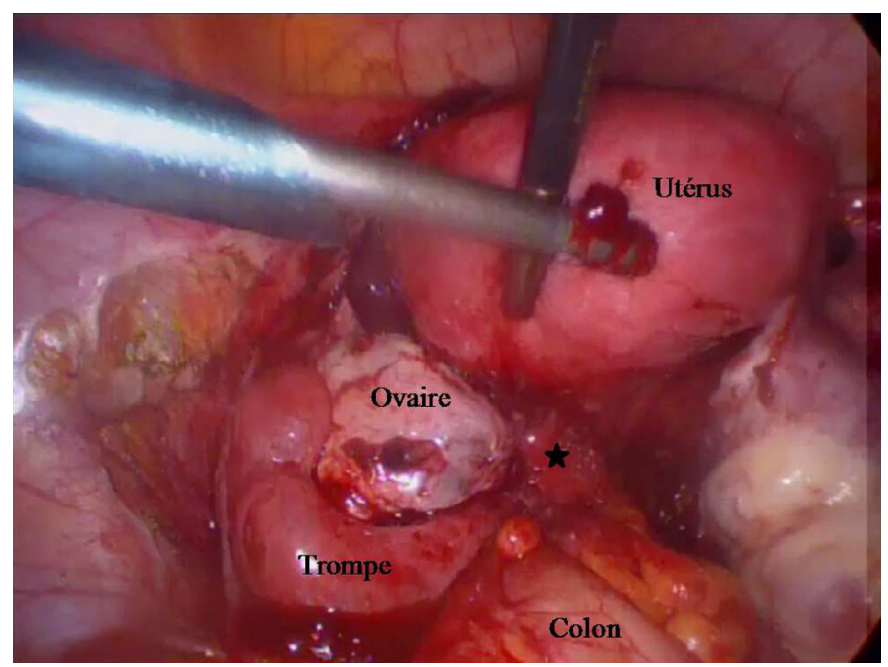

Fig. 3. Aspect cœlioscopique de la lésion d'endométriose utéro-sacrée hyperfixante en TEP- $\left[{ }^{18} \mathrm{~F}\right] \mathrm{FES}$ (patiente $\mathrm{n}^{\mathrm{o}} 4$ ) : lésion d'endométriose (étoile noire) rétro-utérine gauche de $2 \mathrm{~cm}$, accolée à la face postérieure de l'utérus, à l'annexe gauche et au côlon sigmoïde.

Coelioscopic aspect of endometriosis lesion with uptake in $\left[{ }^{18} F\right] F E S-P E T$ (patient 4): left retro-uterine endometriosis lesion (black star) of $2 \mathrm{~cm}$ abutting the posterior face of the uterus, left annex and sigmoid colon.
TEP- $\left[{ }^{18} \mathrm{~F}\right] \mathrm{FES}$ sur les images réalisées 7 à 10 jours après l'instauration du traitement hormonal, contrairement aux patientes non répondeuses [16]. Il existe vraisemblablement une corrélation entre l'intensité de fixation de la $\left[{ }^{18} \mathrm{~F}\right] \mathrm{FES}$ et la réponse tumorale. Aucune patiente négative en $\left[{ }^{18} \mathrm{~F}\right] \mathrm{FES}$ après analyse qualitative ou quantitative ne semble répondre aux traitements hormonaux $[10,17]$. En revanche, une fixation élevée de la $\left[{ }^{18} \mathrm{~F}\right] \mathrm{FES}$ sur la TEP pré-thérapeutique semble pouvoir prédire une bonne réponse à l'hormonothérapie $[10,18]$.

Les données de la littérature concernant la place de la TEP$\left[{ }^{18} \mathrm{~F}\right] \mathrm{FES}$ dans les tumeurs utérines sont relativement récentes. L'intensité de captation de la $\left[{ }^{18} \mathrm{~F}\right] \mathrm{FES}$ dépend du degré de différenciation fonctionnelle du tissu, c'est-à-dire de sa densité en RE : plus une lésion utérine est différenciée, plus sa densité en RE et son intensité de fixation en TEP- $\left[{ }^{18}\right.$ F]FES seront élevées. En 2008, Tsujikawa et al. ont étudié 38 patientes présentant des tumeurs utérines bénignes ou malignes en $\left[{ }^{18} \mathrm{~F}\right] \mathrm{FDG}$ et $\left[{ }^{18} \mathrm{~F}\right] \mathrm{FES}$. Les patientes avec adénocarcinome endométrioïde présentaient des SUV moyens plus élevés en $\left[{ }^{18} \mathrm{~F}\right] \mathrm{FDG}(9,6 \pm 3,3)$ qu'en $\left[{ }^{18} \mathrm{~F}\right] \mathrm{FES}(3,8 \pm 1,8 ; p<005)$, alors que les patientes avec hyperplasie endométriale ou léiomyome présentaient des SUV moyens plus élevés en $\left[{ }^{18}\right.$ F]FES (respectivement $7,0 \pm 2,9$ et $4,2 \pm 2,4$ ) qu'en 

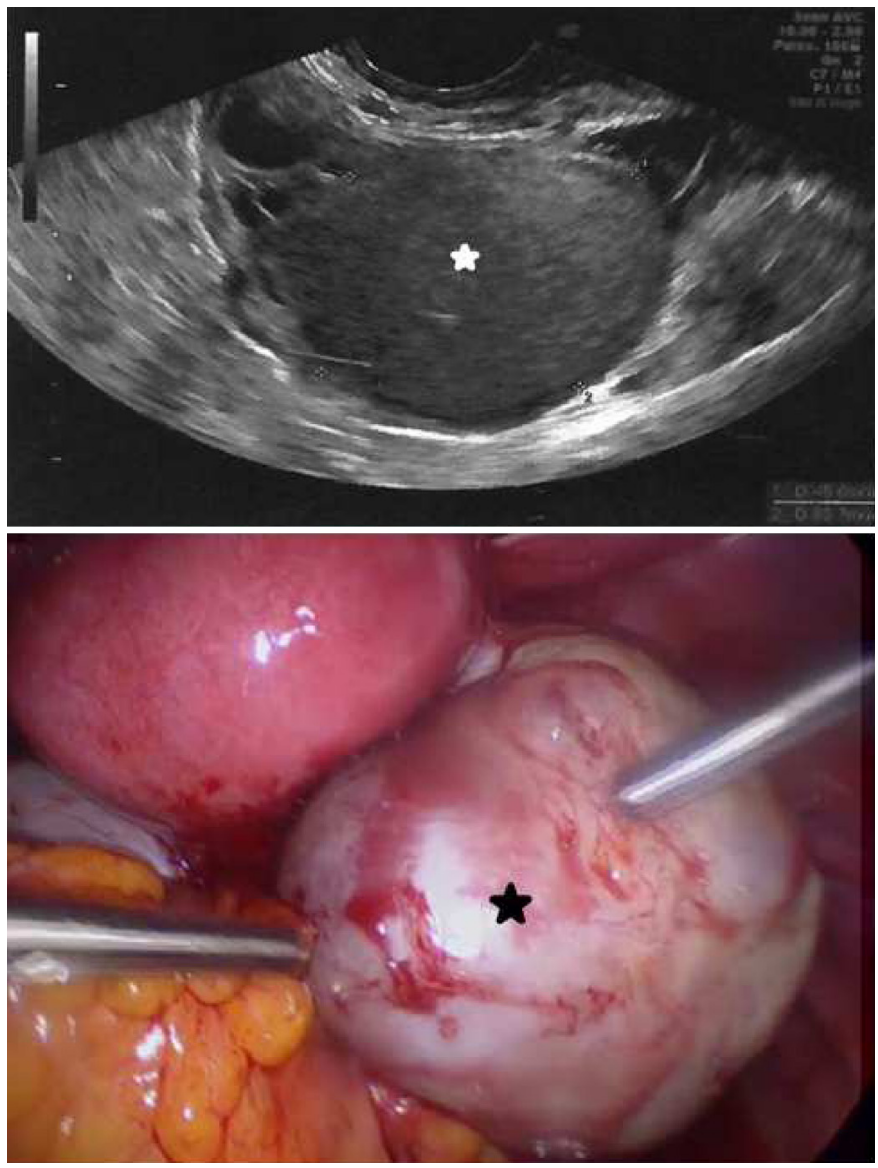

Fig. 4. Aspect cœlioscopique (étoile noire) et échographique (étoile blanche) de l'endométriome de la patiente $\mathrm{n}^{\circ} 4$ : endométriome de $6 \mathrm{~cm}$ de l'ovaire droit, au contact de l'utérus.

Coelioscopic (black star) and echographic (white star) aspect of endometrioma from patient 4: $6 \mathrm{~cm}$-endometrioma of the right ovary, in contact with the uterus.

$\left[{ }^{18} \mathrm{~F}\right] \mathrm{FDG}$ (respectivement, $1,7 \pm 0,3$ et $2,2 \pm 1,1 ; p<0,05$ ). La captation du traceur chez les patientes avec adénocarcinome endométrioïde était significativement plus élevée en [18F]FDG et plus faible en $\left[{ }^{18} \mathrm{~F}\right] \mathrm{FES}$ par rapport aux patientes avec hyperplasie de l'endomètre. Chez les patientes avec sarcome utérin, la fixation était significativement plus élevée en $\left[{ }^{18} \mathrm{~F}\right] \mathrm{FDG}$ et plus faible en $\left[{ }^{18} \mathrm{~F}\right] \mathrm{FES}$ comparativement aux patientes avec léiomyome. Ainsi, les deux traceurs, $\left[{ }^{18} \mathrm{~F}\right] \mathrm{FDG}$ et $\left[{ }^{18} \mathrm{~F}\right] \mathrm{FES}$, apportaient des informations physiopathologiques complémentaires pouvant orienter le diagnostic différentiel des tumeurs utérines [11].

L'endométriose est une affection gynécologique fréquente et complexe qui touche 6 à $10 \%$ des femmes en âge de procréer [19]. Elle est définie comme l'implantation de tissu endométrial en dehors de la cavité utérine. Le pelvis est le site le plus fréquemment touché, même si quasiment tous les organes peuvent être atteints. L'endométriose peut être asymptomatique et être découverte de manière fortuite. Elle peut aussi être responsable de symptômes variés incluant dysménorrhée, dyspareunie, douleurs pelviennes chroniques parfois très invalidantes et infertilité, avec des répercussions psychologiques et sociales importantes. Le délai entre le début des symptômes et le diagnostic est souvent de plusieurs années. Selon l'hypothèse la plus largement acceptée, l'endométriose proviendrait initialement de l'implantation péritonéale de tissu endométrial reflué de manière rétrograde par les trompes en période menstruelle, mais sa physiopathologie exacte reste mal connue. En cas d'endométriose douloureuse, un traitement hormonal visant à l'obtention d'une aménorrhée (contraceptifs œstroprogestatifs en continu, progestatifs, danazol ou analogues de la $\mathrm{Gn}-\mathrm{RH}$ ) et/ou un traitement chirurgical est proposé, mais les récidives sont possibles, plus fréquentes lors de prises en charge tardives et de chirurgie incomplète [20]. Le retard diagnostique s'explique par l'absence de symptomatologie pathognomonique, le manque de sensibilité de l'examen clinique, normal dans plus de $40 \%$ des cas et les limites des examens complémentaires, échographie pelvienne endovaginale et IRM. À l'heure actuelle, le diagnostic définitif d'endométriose et l'évaluation de son extension lésionnelle nécessitent la réalisation d'une cœlioscopie avec biopsie et analyse histologique dans la mesure du possible d'au moins une zone pathologique [20]. Les contraintes imposées par cette exploration chirurgicale peuvent participer, notamment chez les patientes jeunes, au retard de sa réalisation et ainsi au retard diagnostique. Une nouvelle technique d'imagerie permettant de détecter correctement et de manière non invasive les lésions d'endométriose permettrait de poser le diagnostic plus précocement et d'éviter l'exploration chirurgicale dans certains cas. Les lésions d'endométriose, dont le développement et la croissance sont œstrogéno-dépendants, expriment des RE : ce sont donc des lésions bénignes qui pourraient potentiellement être détectées en TEP- $\left[{ }^{18} \mathrm{~F}\right] \mathrm{FES}$.

$\mathrm{La}\left[{ }^{18} \mathrm{~F}\right] \mathrm{FES}$ n'ayant pas d'AMM en France, la première phase du projet ENDOTEP a notamment été consacrée à la rédaction des documents administratifs, la mise au point de la radiosynthèse, les études de toxicologie avant l'obtention des autorisations par les autorités de régulation française ANSM, ASN et CPP, pour la production de la $\left[{ }^{18} \mathrm{~F}\right] \mathrm{FES}$ uniquement dans le cadre de l'essai ENDOTEP par l'industriel Cyclopharma dans un de ses sites de production autorisé lui-même pour cette recherche. Ceci explique le délai entre l'obtention du financement du PHRC en 2010 et les premiers résultats que nous rapportons dans cet article.

À notre connaissance, la TEP- $\left[{ }^{18} \mathrm{~F}\right] \mathrm{FES}$ n'a jamais été étudiée jusqu'à présent dans l'endométriose. Notre objectif principal avec ce PHRC interrégional repose sur l'hypothèse que la TEP- $\left[{ }^{18} \mathrm{~F}\right] \mathrm{FES}$ reflétant la richesse des tissus en RE, elle pourrait permettre d'identifier des lésions d'endométriose qui, par définition, correspondent à du tissu endométrioïde (naturellement riche en RE) implanté en dehors de la cavité utérine. Une seule des quatre patientes a présenté une lésion d'endométriose en TEP- $\left[{ }^{18} \mathrm{~F}\right] \mathrm{FES}$, confirmée en cœlioscopie par un aspect macroscopique typique, mais non détectée par l'échographie endovaginale pré-opératoire. L'échographie pelvienne est un examen complémentaire de première intention en cas de suspicion d'endométriose, du fait de son accessibilité et de sa facilité de réalisation, mais elle présente une sensibilité variant entre 10 et $90 \%$ en fonction de la localisation anatomique des lésions et est, en général, moins performante 
pour détecter les lésions profondes que superficielles [21]. Les lésions superficielles ont une infiltration en profondeur n'excédant pas 2 ou $3 \mathrm{~mm}$ à la surface du péritoine, de la séreuse des organes pelviens (utérus, vagin, rectum, sigmoïde, vessie, trompes) ou de la corticale ovarienne. Les lésions profondes sont sous-péritonéales caractérisées par une pénétration dans l'espace rétropéritonéal et/ou dans la paroi des organes pelviens (vessie, uretère, rectum, vagin, ligaments utéro-sacrés. . .) à une profondeur d'au moins $5 \mathrm{~mm}$ [22]. Dans notre étude, l'IRM a également détecté des lésions d'endométriose utéro-sacrées, non confirmées par l'examen cœlioscopique de référence et, inversement, n'a pas détecté certaines lésions prouvées en cœlioscopie. Considérée comme la technique d'imagerie de référence pour le diagnostic d'endométriose profonde car elle permet d'avoir une vue complète du pelvis, l'IRM est habituellement plus performante que l'échographie pelvienne. Elle a cependant une sensibilité très variable, estimée entre 23 et $89 \%$ en fonction des études et surtout de la localisation anatomique des lésions [23]. La TEP$\left[{ }^{18} \mathrm{~F}\right] \mathrm{FES}$ pourrait être intéressante dans la détection des lésions d'endométriose profonde, pathologie où l'imagerie morphologique n'apparaît pas optimale. Enfin, si on se réfère à l'objectif principal de l'étude qui est de faire le diagnostic d'endométriose, la fixation d'une seule lésion est a priori suffisante car spécifique de tissus riches en RE. La TEP- $\left[{ }^{18}\right.$ F]FES pourrait alors être utilisée comme un outil diagnostique non invasif plus spécifique que l'échographie et l'IRM.

Aucune lésion d'endométriose n'a été visualisée en TEP$\left[{ }^{18} \mathrm{~F}\right] \mathrm{FES}$ chez les patientes en aménorrhée thérapeutique car recevant un traitement hormonal en continu. Chez ces mêmes patientes, on observe une fixation physiologique de l'endomètre plus faible que chez la patiente en $1^{\text {re }}$ partie de cycle (patiente $\mathrm{n}^{\mathrm{O}} 4$ ), en rapport avec la mise au repos de leur endomètre qui s'atrophie sous traitement hormonal et exprime moins de RE. La fixation physiologique de l'endomètre, supérieure à celle du myomètre, a déjà été étudiée dans la littérature. Dans l'étude de Tsuchida et al., la fixation de l'endomètre était significativement supérieure en première partie de cycle qu'en seconde partie (SUV $=6,0 \pm 1,0$ en phase proliférative vs $4,0 \pm 1,3$ en phase sécrétoire; $p=0,022$ ) et dépendait du taux d'œstrogènes circulants [24]. Les lésions d'endométriose des patientes sous traitement hormonal subissent probablement le même sort que l'endomètre mis au repos et une modulation de l'expression tissulaire en RE pourrait expliquer l'absence de visualisation des lésions d'endométriose chez ces trois patientes. L'analyse immunohistochimique de ces lésions d'endométriose était globalement hétérogène entre les patientes ( 0 à $80 \%$ ), mais aussi entre les lésions d'une même patiente. Il faut rappeler que l'analyse immunohistochimique n'identifiait que les RE $\alpha$ et non les RE $\beta$. Cette hétérogénéité de l'expression des sous-types de RE pourrait être rapprochée de ce qui a été rapporté dans les endomètres atrophiques (patientes ménopausées traitées par tamoxifène), où il a été mis en évidence des quantités de RE similaires à celles trouvées chez des patientes non traitées. mais avec une proportion de REß plus importante que celle de RE $\alpha$ [25]. Cette explication pourrait donc aussi être reprise pour notre étude, le traitement œstroprogestatif ayant pu aussi conduire à une modulation de l'activation/expression des soustypes de RE. Enfin, l'absence de visualisation en TEP- $\left[{ }^{18} \mathrm{~F}\right] \mathrm{FES}$ des foyers exprimant des RE pourrait aussi s'expliquer en partie par le fait que les plus volumineux foyers d'endométriose positifs aux RE restaient de taille millimétrique. Deux lésions ovariennes kystiques (endométriomes) ont été visualisées sous la forme de volumineuses lésions hypofixantes en TEP$\left[{ }^{18} \mathrm{~F}\right]$ FES, ne présentant pas de franc rehaussement de fixation périphérique : ces lésions kystiques, majoritairement liquidiennes, présentent probablement une composante tissulaire $\mathrm{RE}+$ (paroi kystique) trop faible pour être nettement visualisée en TEP- $\left[{ }^{18}\right.$ F]FES (effet de volume partiel), même si l'analyse immunohistochimique retrouvait une expression de RE. L'acquisition TDM (non injectée) couplée à la TEP n'a pas non plus permis de repérer les lésions d'endométriose pelvienne, en dehors cependant des lésions hypodenses kystiques (endométriomes). Le scanner n'est habituellement pas indiqué dans les pathologiques gynécologiques pelviennes, car bien moins performant que l'IRM dans cette indication [23]. La fusion d'images TEP et IRM pourrait permettre de pallier les insuffisances du scanner de repérage dans cette indication. Nos premiers résultats montrant que la TEP- $\left[{ }^{18}\right.$ F]FES ne détecte pas les lésions d'endométriose chez les patientes en aménorrhée thérapeutique vont nous conduire à préciser dans les critères d'inclusion la nécessité de ne pas avoir pris ou d'arrêter tout traitement hormonal entre 1 à 3 mois précédents la TEP$\left[{ }^{18} \mathrm{~F}\right] \mathrm{FES}$. Ces résultats incitent également à réaliser la TEP$\left[{ }^{18} \mathrm{~F}\right] \mathrm{FES}$ chez les patientes en première partie de cycle, période où l'expression endométriale des RE apparaît maximale et où les chances de détecter les lésions d'endométriose apparaissent optimales. En effet, il existe une variation cyclique de l'expression des RE sous la dépendance des œstrogènes pendant la période de la reproduction, l'endomètre subit des modifications morphologiques et physiologiques caractérisées par une croissance (phase proliférative, première partie du cycle), une différenciation sécrétoire (seconde partie) et, en l'absence de fécondation, une menstruation et une régénération. Lors de la première partie du cycle menstruel, les œstrogènes se fixent sur les récepteurs RE, ce qui conduit à la production de différentes protéines dont les RE eux-mêmes et les récepteurs à la progestérone (préparant ainsi la seconde partie du cycle). L'activation des RE par les œstrogènes conduit également à l'activation de voie signalitique, notamment de prolifération cellulaire (responsable également de la prolifération des lésions d'endométriose). Les RE diminuent ensuite puis disparaissent pendant la phase sécrétoire à la fois dans l'épithélium et le stroma [25].

Plusieurs limites techniques de l'imagerie TEP- $\left[{ }^{18} \mathrm{~F}\right] \mathrm{FES}$ peuvent être soulevées à travers ces quatre premiers cas. Tout d'abord, l'interprétation des images à l'étage abdominal peut être rendue difficile en raison d'une importante fixation digestive de la $\left[{ }^{18} \mathrm{~F}\right] \mathrm{FES}$. En effet, le traceur injecté est rapidement extrait de la circulation sanguine par le foie, où il est métabolisé. Il est ensuite excrété par les voies biliaires dans l'intestin grêle où se produit une réabsorption intestinale avant d'être éliminé par voie urinaire. Cette biodistribution de la 
$\left[{ }^{18} \mathrm{~F}\right] \mathrm{FES}$ est à l'origine d'une activité hépato-biliaire pouvant être associée à des fixations intestinales particulièrement intenses (SUV max moyen évalué respectivement à $18,24 \pm 2,15,216,20 \pm 62,67$ et 27,56 $\pm 15,29$ au niveau du foie, de la vésicule biliaire et de l'intestin grêle). La clairance de la $\left[{ }^{18} \mathrm{~F}\right] \mathrm{FES}$ est rapide et seuls $20 \%$ du traceur ne sont pas métabolisés $20 \mathrm{~min}$ après l'injection, les métabolites radiomarqués étant responsables d'un bruit de fond relativement constant et homogène sur les images après ces 20 premières minutes. Ainsi, le début des acquisitions rapidement après ou en même temps que l'injection permettrait une bonne visualisation des tissus riches en RE [4]. Le jeûne de 6 h préconisé chez nos patientes afin de diminuer l'excrétion hépato-biliaire et intestinale de la $\left[{ }^{18} \mathrm{~F}\right] \mathrm{FES}$ n'a pas permis de s'affranchir des fixations intestinales. En revanche, la réalisation d'images précoces à 20 min puis tardives à 60 min a permis de s'assurer de l'origine digestive physiologique des fixations abdominales lorsqu'elles se déplaçaient entre les deux séries d'images. L'acquisition dynamique précoce semblait également intéressante car aucune fixation digestive pelvienne n'était visualisée sur ces images réalisées dans les 20 premières minutes suivant l'injection et l'activité urinaire au sein de la vessie était limitée, voire nulle. Enfin, dans les cas que nous rapportons, aucune lésion d'endométriose abdominale n'a été mise en évidence en cœlioscopie.

Différents analogues fluorés de l'œstradiol ont été étudiés et pourraient concurrencer, voire remplacer, un jour la $\left[{ }^{18} \mathrm{~F}\right] \mathrm{FES}$. Certains n'ont pas connu de développement clinique parce qu'ils présentaient une affinité pour les RE inférieure à celle de la $\left[{ }^{18} \mathrm{~F}\right] \mathrm{FES}$ ou une affinité trop faible pour la sex hormone binding globulin (SHBG), protéine de transport permettant le passage intracellulaire de l'œstradiol et de ses analogues fluorés [26]. En revanche, les difluoro-œstradiols ont été décrits comme plus stables in vivo que la $\left[{ }^{18} \mathrm{~F}\right] \mathrm{FES}$ car l'adjonction d'un second atome de fluor-18 en position 2- ou 4- de la molécule de $\left[{ }^{18} \mathrm{~F}\right] \mathrm{FES}$ diminue leur catabolisme [27]. L'un d'eux, le 4fluoro-11beta-methoxy-16alpha-18F-fluoroœstradiol

(4FMFES), a prouvé son efficacité préclinique : il présenterait une captation utérine plus élevée que la $\left[{ }^{18} \mathrm{~F}\right] \mathrm{FES}$ chez la femelle rat immature et un meilleur contraste, ainsi qu'une captation par les tumeurs RE+ plus élevée que la $\left[{ }^{18} \mathrm{~F}\right] \mathrm{FES}$ chez la souris [28,29]. Beauregard et al. ont publié en 2009 la première étude clinique de phase I évaluant la biodistribution du 4FMFES et démontrant une bonne captation utérine du traceur et l'absence d'effets indésirables chez 10 femmes saines [30]. L'efficacité clinique de ce nouveau traceur des RE reste à démontrer chez des patientes avec tumeurs RE+.

\section{Conclusion}

Dans cet article, nous rapportons le premier cas de fixation de $\left[{ }^{18} \mathrm{~F}\right]$ FES par une lésion d'endométriose (patiente $\mathrm{N}^{\circ} 4$ ). Chez les trois premières patientes, en aménorrhée thérapeutique lors de la TEP- $\left[{ }^{18} \mathrm{~F}\right] \mathrm{FES}$, les lésions d'endométriose n' ont pas été détectées, probablement du fait d'une modulation de l'expression des RE sous traitement hormonal. Ces premiers résultats incitent à réaliser les examens en première partie de cycle menstruel et après sevrage en traitement hormonal. La production de $\left[{ }^{18} \mathrm{~F}\right] \mathrm{FES}$ dans le cadre du PHRC ENDOTEP est autorisée depuis le second semestre 2013 et le PHRC va maintenant pouvoir ouvrir les autres centres investigateurs.

\section{Déclaration d'intérêts}

Les auteurs déclarent ne pas avoir de conflits d'intérêts en relation avec cet article.

\section{Références}

[1] Jensen EV, Jordan VC. The estrogen receptor: a model for molecular medicine. Clin Cancer Res 2003;96:1980-9.

[2] Mintun MA, Welch MJ, Siegel BA, Mathias CJ, Brodack JW, McGuire $\mathrm{AH}$, et al. Breast cancer: PET imaging of estrogen receptors. Radiology 1988;169:45-8.

[3] Peterson LM, Mankoff DA, Lawton T, Yagle K, Schubert EK, Stekhova S, et al. Quantitative imaging of estrogen receptor expression in breast cancer with PET and 18F-fluoroestradiol. J Nucl Med 2008;49:367-74.

[4] Sundararajan L, Linden HM, Link JM, Krohn KA, Mankoff DA. 18Ffluoroestradiol. Semin Nucl Med 2007;37:470-6.

[5] Kostakoglu L. Radionuclide response assessment of breast cancer. Semin Nucl Med 2013;43:299-316.

[6] Linden HM, Dehdashti F. Novel methods and tracers for breast cancer imaging. Semin Nucl Med 2013;43:324-9.

[7] McGuire AH, Dehdashti F, Siegel BA, Lyss AP, Brodack JW, Mathias CJ, et al. Positron tomographic assessment of 16 alpha-[18F] fluoro-17 betaestradiol uptake in metastatic breast carcinoma. J Nucl Med 1991;32: 1526-31.

[8] Peterson LM, Kurland BF, Link JM, Schubert EK, Stekhova S, Linden $\mathrm{HM}$, et al. Factors influencing the uptake of $18 \mathrm{~F}$-fluoroestradiol in patients with estrogen receptor positive breast cancer. Nucl Med Biol 2011;38:969-78.

[9] Dehdashti F, Mortimer JE, Siegel BA, Griffeth LK, Bonasera TJ, Fusselman MJ, et al. Positron tomographic assessment of estrogen receptors in breast cancer: comparison with FDG-PET and in vitro receptor assays. J Nucl Med 1995;36:1766-74.

[10] Linden HM, Stekhova SA, Link JM, Gralow JR, Livingston RB, Ellis GK, et al. Quantitative fluoroestradiol positron emission tomography imaging predicts response to endocrine treatment in breast cancer. J Clin Oncol 2006;24:2793-9.

[11] Tsujikawa T, Yoshida Y, Mori T, Kurokawa T, Fujibayashi Y, Kotsuji F, et al. Uterine tumors: pathophysiologic imaging with 16alpha-[18F] fluoro-17beta-estradiol and 18F fluorodeoxyglucose PET - initial experience. Radiology 2008;248:599-605.

[12] Moresco RM, Scheithauer BW, Lucignani G, Lombardi D, Rocca A, Losa $\mathrm{M}$, et al. Oestrogen receptors in meningiomas: a correlative PET and immunohistochemical study. Nucl Med Commun 1997;18:606-15.

[13] Yoshida Y, Kurokawa T, Tsujikawa T, Okazawa H, Kotsuji F. Positron emission tomography in ovarian cancer: $18 \mathrm{~F}$-deoxy-glucose and 16alpha18F-fluoro-17beta-estradiol PET. J Ovarian Res 2009;2:7. http:// dx.doi.org/10.1186/1757-2215-2-7.

[14] Mortimer JE, Dehdashti F, Siegel BA, Katzenellenbogen JA, Fracasso P, Welch MJ. Positron emission tomography with 2-[18F]fluoro-2-deoxy-Dglucose and 16alpha-[18F]fluoro-17beta-estradiol in breast cancer: correlation with estrogen receptor status and response to systemic therapy. Clin Cancer Res 1996;2:933-9.

[15] Yang Z, Sun Y, Zhang Y, Xue J, Wang M, Shi W, et al. Can fluorine18 fluoroestradiol positron emission tomography-computed tomography demonstrate the heterogeneity of breast cancer in vivo? Clin Breast Cancer 2013;13:359-63.

[16] Dehdashti F, Flanagan FL, Mortimer JE, Katzenellenbogen JA, Welch MJ, Siegel BA. Positron emission tomographic assessment of "metabolic flare" to predict response of metastatic breast cancer to antiestrogen therapy. Eur J Nucl Med 1999;26:51-6. 
[17] Mortimer JE, Dehdashti F, Siegel BA, Trinkaus K, Katzenellenbogen JA, Welch MJ. Metabolic flare: indicator of hormone responsiveness in advanced breast cancer. J Clin Oncol 2001;19:2797-803.

[18] Dehdashti F, Mortimer JE, Trinkaus K, Naughton MJ, Ellis M, Katzenellenbogen JA, et al. PET-based estradiol challenge as a predictive biomarker of response to endocrine therapy in women with estrogenreceptor-positive breast cancer. Breast Cancer Res Treat 2009;113: 509-17.

[19] Giudice LC. Clinical practice. Endometriosis. N Engl J Med 2010;362: 2389-98.

[20] Poncelet C, Ducarme G. Endometriosis: good practice rules for diagnostic laparoscopy. J Gynecol Obstet Biol Reprod (Paris) 2007;36:135-40.

[21] Bazot M, Lafont C, Rouzier R, Roseau G, Thomassin-Naggara I, Daraï E. Diagnostic accuracy of physical examination, transvaginal sonography, rectal endoscopic sonography, and magnetic resonance imaging to diagnose deep infiltrating endometriosis. Fertil Steril 2009;92:1825-33.

[22] Fritel X. Endometriosis anatomoclinical entities. J Gynecol Obstet Biol Reprod (Paris) 2007;36:113-8.

[23] Jarlot C, Anglade E, Paillocher N, Moreau D, Catala L, Aubé C. MR imaging features of deep pelvic endometriosis: correlation with laparoscopy. J Radiol 2008;89:1745-54.

[24] Tsuchida T, Okazawa H, Mori T, Kobayashi M, Yoshida Y, Fujibayashi Y, et al. In vivo imaging of estrogen receptor concentration in the endometrium and myometrium using FES PET - influence of menstrual cycle and endogenous estrogen level. Nucl Med Biol 2007;34:205-10.

[25] Bergeron C. Effect of oestrogens and antiestrogens on the endometrium. Gynecol Obstet Fertil 2002;30:933-7.

[26] Couturier O, Luxen A, Chatal JF, Vuillez JP, Rigo P, Hustinx R. Fluorinated tracers for imaging cancer with positron emission tomography. Eur J Nucl Med Mol Imaging 2004;31:1182-206.

[27] Benard F, Ahmed N, Beauregard JM, Rousseau J, Aliaga A, Dubuc C, et al. [18F]fluorinated estradiol derivatives for oestrogen receptor imaging: impact of substituents, formulation and specific activity on the biodistribution in breast tumour-bearing mice. Eur J Nucl Med Mol Imaging 2008;35:1473-9.

[28] Seimbille Y, Rousseau J, Benard F, Morin C, Ali H, Avvakumov G, et al. 18F-labeled difluoroestradiols: preparation and preclinical evaluation as estrogen receptor-binding radiopharmaceuticals. Steroids 2002;67:765-75.

[29] Paquette M, Phoenix S, Ouellet R, Langlois R, van Lier JE, Turcotte EE, et al. Assessment of the novel estrogen receptor PET tracer 4-fluoro11beta-methoxy-16alpha-[F]fluoroestradiol (4FMFES) by PET imaging in a breast cancer murine model. Mol Imaging Biol 2013;15:625-32.

[30] Beauregard JM, Croteau E, Ahmed N, van Lier JE, Benard F. Assessment of human biodistribution and dosimetry of 4-fluoro-11beta-methoxy16alpha-18F-fluoroestradiol using serial whole-body PET/CT. J Nucl Med 2009;50:100-7. 